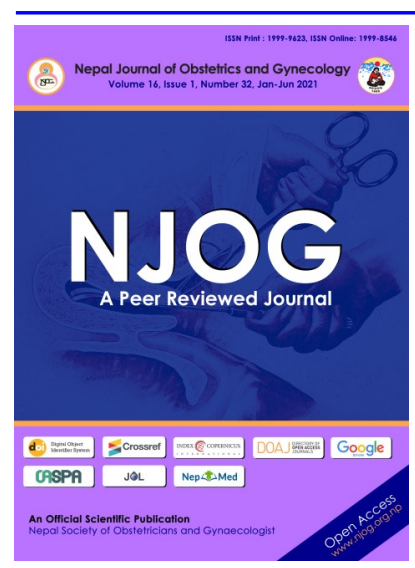

CORRESPONDENCE

Dr Ayeshna Gurung

Department of Radiology and Imaging

Nobel Medical College and Teaching Hospital, Biratnagar, Nepal

E-mail:

ayeshna@gamil.com; Mobile: +977-9843671781

Received: June 7, 2021

Accepted: June 15, 2021

\section{Citation:}

Gurung A, Baral R, Koirala B, Shrestha SK. Association of laboratory parameters with chest $\mathrm{CT}$ findings in COVID-19 patients. Nep J Obstet Gynecol. 2021;16(32):115119. DOI: https:// doi.org/10.3126/ njog.v16i1.37915

\title{
Association of laboratory parameters with chest CT findings in COVID-19 patients
}

\author{
Ayeshna Gurung ${ }^{1}$, Reetu Baral ${ }^{2}$, BinitKoirala ${ }^{1}$, Suman Kumar Shrestha ${ }^{1}$ \\ ${ }^{1}$ Department of Radiology and Imaging, ${ }^{2}$ Department of Pathology \\ Nobel Medical College and Teaching Hospital, Biratnagar, Nepal
}

\section{ABSTRACT}

Aims: To analyze the Chest CT Scan and association of the severity score with the serum biomarkers D-dimer, C-reactive protein (CRP), Lactate dehydrogenase $(\mathrm{LDH})$ and Ferritin levels.

Methods: This is a retrospective study done from the database of Department of Radiology and Department of Pathology at Nobel medical college and teaching hospital during the peak of the second wave of the pandemic from $1^{\text {st }}$ April 2021 to $31^{\text {st }}$ May 2021. Data for the Chest CT scan and lab parameters were analyzed and correlated. Ethical approval was obtained from the Institutional review committee of Nobel Medical College and Teaching Hospital. Descriptive statistical analysis was performed.

Results: A total of 263 cases of Chest CT scan were done in 2 months period who were diagnosed as cases of COVID 19. In this study the severity and scores were taken from the database in the Radiology department. The lab parameters like D-dimer, CRP, LDH and Ferritin levels were studied from the database in the lab. Age of the patients ranged from 19 to 89 years with maximum cases $68(25.8 \%)$ seen in the age group of 50-59 years. According to the chest CT Scan the severity score was in between $11-15$ in $92(48.3 \%)$ cases. Fifty six cases showed abnormal levels of D-dimer, Ferritin, CRP and LDH. Ddimer was raised in 26 (46.4\%), LDH was raised in 48 (87.2\%) while Ferritin and CRP was also raised in all 56 cases.

Conclusions: There was a positive correlation between the biomarkers and the Chest CT severity score. The severity of the disease with involvement of the lungs can be estimated by correlating with the lab parameters.

Keywords: COVID 19, Ferritin level, CT Severity score

\section{INTRODUCTION}

After the outbreak of SARS-Cov2 from China in December 2019 it has made a major impact on global health. There has been an overwhelming second wave of the COVID-19 pandemic in Nepal with new daily positive cases reaching upto 9238 on $13^{\text {th }}$ May 2021 and exhausting the health care system.

Severe pneumonia, fever, fatigue, dry cough and respiratory distress are the major symptoms of patients presenting in the hospital. ${ }^{1}$ Clinical information along with laboratory data, Chest x-ray and chest CT scans are implemented in COVID-19 pandemic as part of the diagnostic workup for patient prognosis and also clinicalmanagement. ${ }^{2}$ Recognizing the laboratory parameters that can aid in identifying severe and non-severe cases or those at high or low risk of mortality can help in clinical assessment. ${ }^{3}$

Bilateral ground glass opacities (GGOs) and consolidation having predominantly peripheral distribution, crazy-paving pattern, pulmonary artery enlargement and reverse halo sign are some of the common findings of COVID- 19 on high resolution CT. ${ }^{4-7}$ The advantage of chest CT is not just the initial disease severity assessment but also as a progression comparisontool defined by area of involvement of the entire lung. ${ }^{8-10}$ The CT severity score index has been developed and implemented in reporting. It is a scoring system used to assess lung involvement based on appr- 
oximate estimation of pulmonary involved areas. ${ }^{6}$ Inpatients with moderate and severe symptoms severity scoring can improve their sensitivity for diagnosing COVID-19 pneumonia. $^{8} \mathrm{CT}$ pulmonary angiography is considered as a diagnostic aid in patients with complications where pulmonary embolism is suspected. ${ }^{11}$

Higher lung severity score is associated with altered laboratory tests. Correlation has been seen between altered lymphocytopenia, D-dimer, LDH, CRP and fibrinogen tests and severity of the lungs involvement on chest CT. ${ }^{12}$

This study aims to assess the association of deranged laboratory parameters of COVID-19 patients with severity of lung involvement as seen on chest CT.

\section{METHODS}

This was a retrospective hospital based descriptive cross-sectional study conducted during the period from $1^{\text {st }}$ April 2021 to $31^{\text {st }}$ May 2021 in the Department of Radiology and Department of Pathology in Nobel Medical College and Teaching Hospital, Biratnagar, Nepal. CT scan reports of patients who were diagnosed as COVID 19 cases were extracted from the database in the Department of Radiology. All patients who were COVID RT-PCR positive and undergone CT Scan chest in Nobel medical college teaching hospital were included in the study.A semi quantitative scoring system was used for chest CT severity score. ${ }^{7,13}$ It was graded according to the percentage of each lobar involvement of 5 lobes with GGOs, consolidation, crazy paving on the basis of the area involved as: 0 (no involvement), 1 ( $<5 \%$ involvement), 2 (5-25\% involvement), 3 (26$50 \%$ involvement), 4 (51-75\% involvement) and 5 ( $>75 \%$ involvement) with total scoring done out of 25. [Figure-1]

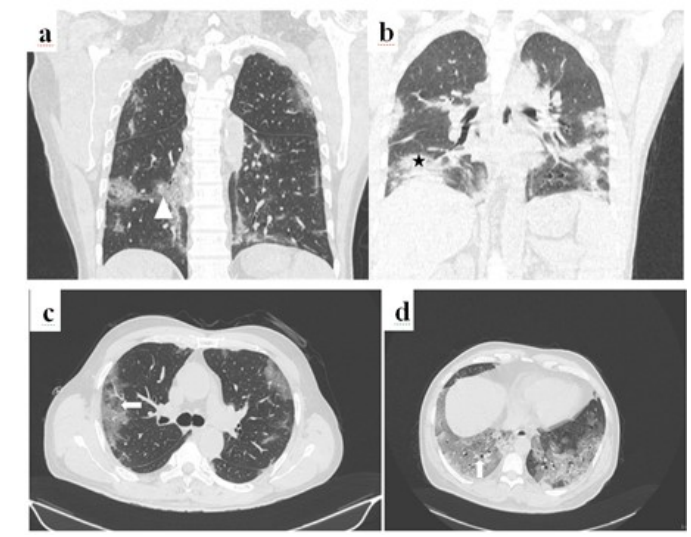

Figure-1: Coronal chest CT scan showing the scoring system used to evaluate the degree of parenchyma involvement: Crazy paving (arrow head) in a patient with total severity score of $14 / 25$ (a), Consolidations (star) in a patient with severity score of $18 / 25$. Axial chest CT scan showing ground glass opacities (arrows) in a patient with severity score of 12/25 (c) and severity score of 21/25 (d)
A database search was done in the lab with the hospital number of the patients who had undergone CT Scan for lab tests like C-reactive protein (CRP), lactate dehydrogenase (LDH), Ferritin and D-dimer. The results of the lab tests and the severity score from the CT Scan chest were analyzed in SPSS 20.0v.

\section{RESULTS}

A total of 263 cases of COVID RT PCR positive patients had CT scan chest done in the Department of Radiology between $1^{\text {st }}$ April 2021 and $31^{\text {st }}$ May 2021. These two months were the peak of the second wave of the pandemic in Nepal. The mean age of the patients in this study was $47.8 \pm 14.4$ years (range 19-86) and median of 48 years. It was found that gender distribution in this study is Male (74\%) and Female (26\%). [Table-1]

Table-1: Age distribution of the study population $(\mathrm{N}=263)$

\begin{tabular}{ccc}
\hline Age in years & $\mathrm{N}$ & $\%$ \\
\hline$<20$ & 3 & 1.1 \\
$20-29$ & 23 & 8.7 \\
$30-39$ & 63 & 23.9 \\
$40-49$ & 46 & 17.4 \\
$50-59$ & 68 & 25.8 \\
6069 & 37 & 14.0 \\
$70-79$ & 20 & 7.6 \\
$80-89$ & 3 & 1.1 \\
\hline
\end{tabular}

The mean chest CT severity score of the patients in this study was $13.5 \pm 5$ and mode was 12 with maximum number (92) having severity score between 11 and 15 .

Table-2: Chest CT severity score distribution $(\mathrm{N}=263)$

\begin{tabular}{ccc}
\hline CT severity score & $\mathbf{N}$ & $\mathbf{\%}$ \\
\hline $1-5$ & 14 & 5.3 \\
$6-10$ & 66 & 25 \\
$11-15$ & 92 & 34 \\
$16-20$ & 62 & 23 \\
$21-25$ & 29 & 11 \\
\hline
\end{tabular}

Four biomarkers (D-dimer, Ferritin, LDH, CRP) were studied in the lab. These biomarkers were routinely sent in lab by the clinicians from COVID OPD, ward and ICU. Out of all the patients who had CT scan done 56 cases had all 4 lab tests done. Analysis and correlation of these were done with the CT scan reports. The D-dimer was high in 26 $(46.4 \%)$, Ferritin was raised in all 56 cases, $\mathrm{LDH}$ was raised in $48(87.2 \%)$ and CRP was raised in $100 \%$ cases. [Table-3]

Table-3: Laboratory parameters

\begin{tabular}{ll}
\hline Biomarker & Median (Range) \\
\hline D-dimer (M/GL) & $0.4(0.2-2.0)$ \\
Ferritin (NG/ML) & $324(29.1-1240)$ \\
LDH (U/L) & $352(150-1100)$ \\
CRP & $24(12-48)$ \\
\hline
\end{tabular}




\section{DISCUSSION}

COVID-19 is a global pandemic with Nepal going through $2^{\text {nd }}$ wave that has been significantly worse in comparison to the $1^{\text {st }}$ wave. Early diagnosis is an essential for management of the patients.

Of the 263 COVID-19 patients studied in this study mean age was 47.8 years (range 19-86). In a study by Shuchang et $\mathrm{al}^{14}$ and Tan $\mathrm{C}$ et $\mathrm{al}^{15}$ mean age was48yrs and $48.89 \pm 18.47$ respectively.

The clinical spectrum of SARS-CoV-2 infection can range from subclinical infections to severe cases of pneumonia with respiratory failure, ARDS. ${ }^{16-17} \mathrm{Ra}-$ diological and laboratory tests work-up are often used to define patient prognosis and to guide clinical management of COVID-19.

Chest CT severity scoring was done on the basis of area involved on each lobe of the lung ( 3 on right and 2 on left). A semi quantitative scoring system was used as described by Chang et $\mathrm{al}^{13}$ and mentioned in previous study of Pan et al. ${ }^{7}$ The maximum number of cases was found to have a score of 12 (24 patients) with most of the patients (92) having score between 11-15. Previous studies have mentioned that patient with total CT scores of 16 points or more in comparison to the patients with less than 16 points, the risk of poor prognosis increased by 6.9 times. $^{14}$

The ability of the laboratory tests (CRP, LDH, Ddimer and Ferritin) to assess severity of COVID-19 has been confirmed by meta-analyses. ${ }^{18}$ Besides the importance of the assessment of the severity of the disease and the prognosis of the patient, the identification of laboratory biomarkers associated with COVID-19 has shed light on pathological mechanisms of the disease. There is strong evidence that dysregulated and excessive cytokines release is responsible for rapid progression and high mortality. ${ }^{19}$ Updated findings however indicate that even in the absence of cytokine storm patients are suffering from severe acute respiratory syndrome which tells us the fact that pathogenesis of COVID 19 maybe more complex. Pro inflammatory proteins like CRP and Ferritin are seen elevated in these patients and correlating with the chest CTscan severity score is similar to our findings. ${ }^{2,20}$

Serum Ferritin level was elevated in all 56 cases of our study which is similar to the studies done by different researchers. ${ }^{21,22}$ Different studies have shown that serum ferritin level is also a predictor of COVID 19 disease. Our study showed that the severity score was highest for patients with increased ferritin level with $26(46.4 \%)$ cases followed by moderate cases $21(37.5 \%)$. This is similar to studies done by Kappertet al. ${ }^{23}$ These observations as well as other studies suggested that serum ferritin can be taken as a predictor of COVID 19 severity.
Hyperferritinemic syndromes so far comprises of four clinical entities, septic shock, macrophage activation syndrome (MAS), Still's disease in adults (AOSD) and catastrophic antiphospholipid syndrome (CAPS). All these diseases are characterized by both an extremely elevated serum ferritin level and a partly life-threatening hyperinflammation. The hyperinflammation in patients with COVID 19 along with elevated level of Ferritin shows the similarities between this and the "hyperferritinemic syndromes". ${ }^{24,25}$

The D-dimer is a relatively small protein fragment that is present in the blood following degradation of blood clots by fibrinolysis. The determination of circulating D-dimer concentrations is a sensitive test in clinical practice to diagnose thrombotic states, including pulmonary embolism and DIC. ${ }^{26}$ In our study the D-dimer was seen to be elevated in $26(46.4 \%)$ cases. Majority of the cases 21 $(37.5 \%)$ with increased D-dimer came in the category of higher score according to severity scoring of the chest CT scan. This finding is similar to a study done in China where they also found that the increasing level of D-dimer correlated with the increasing severity seen in the CT Chest. ${ }^{27}$ In a study done in Nepal by Khatri $\mathrm{P}$ et $\mathrm{al}^{28}$ showed that higher the D-dimer level greater the severity and chances of mortality which correlates with our study. ${ }^{28}$

We studied another biomarker the Lactate Dehydrogenase (LDH) to confirm whether they have any role in severity of the disease since elevated levels have been associated with worse outcomes in patients with other viral infections in the past. ${ }^{29}$ Although LDH has been traditionally used as a marker of cardiac damage since the $1960 \mathrm{~s}$, abnormal values can result from multiple organ injury and decreased oxygenation with upregulation of the glycolytic pathway. ${ }^{30}$ Since LDH is present in lung tissue (isozyme 3), patients with severe COVID-19 infections can be expected to release greater amounts of LDH in the circulation, as a severe form of interstitial pneumonia, often evolving into acute respiratory distress syndrome, is the hallmark of the disease. ${ }^{31}$ In our study LDH was elevated in $48(87.2 \%)$ cases all of which were of higher score in chest CT scan. This was seen in studies done by several researchers. ${ }^{32-34}$

C-reactive protein (CRP) is another valuable bio marker to anticipate the severity of COVID 19 cases. ${ }^{35} \mathrm{C}$-reactive protein can be used in the early diagnosis of pneumonia and patients presenting with severe pneumonia had high CRP level. ${ }^{36}$ Our study showed that all 56 cases had elevated CRP level. The higher range of CRP is seen in cases which are categorized underhigher severity score of chest CT scan which is similar to study done by Tan $\mathrm{C}$ et al. ${ }^{15}$ 


\section{CONCLUSIONS}

The severity score of the Chest CT scan showed association with the elevated biomarkers done in the pathology lab. These parameters will help to judge the status and the prognosis of the patients with COVID 19.

\section{REFERENCES}

1. Kooraki S, Hosseiny M, Myers L, Gholamrezanezhad A. Coronavirus (COVID-19) Outbreak: What the Department of Radiology Should Know. J Am Coll Radiol. 2020;17(4):447-51. doi: 10.1016/j.jacr.2020.02.008 Epub 2020 Feb 19. PMID: 32092296; PMCID: PMC7102595.

2. Canovi S, Besutti G, Bonelli E, Iotti V, Ottone $\mathrm{M}$, AlbertazziL, et al. The association between clinical laboratory data and chest CT findings explains disease severity in a large Italian cohort of COVID-19 patients. BMC Infect Dis. 2021;21 (1):157. doi: 10.1186/s12879-021-05855-9 PMID: 33557778; PMCID: PMC7868898.

3. Henry BM, de Oliveira MHS, Benoit S, Plebani M, Lippi G. Hematologic, biochemical and immune biomarker abnormalities associated with severe illness and mortality in coronavirus disease 2019 (COVID-19): a meta-analysis. Clin Chem Lab Med. 2020;58(7):1021-8. doi: 10.1515/cclm-2020-0369 PMID: 32286245.

4. Besutti G, Giorgi Rossi P, Iotti V, Spaggiari L, Bonacini R, Nitrosi A, et al. Accuracy of CT in a cohort of symptomatic patients with suspected COVID-19 pneumonia during the outbreak peak in Italy. Eur Radiol. 2020;30(12):6818-27. doi: 10.1007/s00330-020-07050-x Epub 2020 Jul 14. PMID: 32666316; PMCID: PMC7358325.

5. Kwee TC, Kwee RM. Chest CT in COVID19:What the Radiologist Needs to Know. Radiographics. 2020;40(7):1848-65. https://doi.org/10.1148/rg.2020200159

6. Francone M, Iafrate F, Masci GM, Coco S, Cilia F, Manganaro, et al. Chest CT score in COVID19 patients: correlation with disease severity and short-term prognosis. Eur Radiol. 2020;30 (12):6808-17. https://doi.org/10.1007/s00330020-07033-y

7. Pan F, Ye T, Sun P, Gui S, Liang B, Li L, Zheng $\mathrm{D}$, et al. Time Course of Lung Changes at Chest CT during Recovery from Coronavirus Disease 2019 (COVID-19). Radiol. 2020;295(3):715-21. doi: 10.1148/radiol.2020200370 Epub 2020 Feb 13. PMID: 32053470; PMCID: PMC7233367.

8. Abdel-Tawab M, Basha MAA, Mohamed IAI, Ibrahim HM, Zaitoun MMA, Elsayed SB, et al. Comparison of the CO-RADS and the RSNA chest CT classification system concerning sensiti vity and reliability for the diagnosis of COVID19 pneumonia. Insights Imaging. 2021;12(1):55. doi: 10.1186/s13244-021-00998-4. PMID: 33913066 ; PMCID: PMC8081002.

9. Fan L, Liu S. CT and COVID-19: Chinese experience and recommendations concerning detection, staging and follow-up. Eur Radiol. 2020;30(9):5214-6. doi:10.1007/s00330-02006898-3

10. Yang R, Li X, Liu H, Zhen Y, Zhang X, Xiong $\mathrm{Q}$, et al. Chest CT Severity Score: An Imaging Tool for Assessing Severe COVID-19. Radiol Cardiothorac Imaging. 2020;2(2):e200047. doi: 10.1148/ryct.2020200047 PMID: 33778560; PMCID: PMC7233443.

11.Jha A, Lohani B, Ghimire RK. Current Imaging Trends in COVID-19 Pneumonia. J Nep Med Assoc. 2020;58(226):444-6. doi:10.31729/ jnma.4992

12. Antonio O, Fulvio G, Silvia R, Santangelo RM. Correlations between chest-CT and laboratory parameters in SARS-CoV-2 pneumonia. Medicine. 2021;100(14):e25310. doi: 10.1097/ MD.0000000000025310

13.Chang YC, Yu CJ, Chang SC, Galvin JR, Liu $\mathrm{HM}$, Hsiao $\mathrm{CH}$, et al. Pulmonary sequelae in convalescent patients after severe acute respiratory syndrome: evaluation with thin-section CT. Radiol. 2005;236(3):1067-75. doi: 10.1148/ radiol.2363040958 Epub 2005 Jul 29. PMID: 16055695.

14.Zhou S, Chen C, Hu Y, Lv W, Ai T, Xia L. Chest CT imaging features and severity scores as biomarkers for prognostic prediction in patients with COVID-19. Ann Transl Med. 2020;8(21):1449. doi:10.21037/atm-20-3421

15. Tan C, Huang Y, Shi F, Tan K, Ma Q, Chen Y, et al C-reactive protein correlates with computed tomographic findings and predicts severe COVID-19 early. J Med Virol. 2020;92:85662.

16. Huang C, Wang Y, Li X, Ren L, Zhao J, Hu Y, et al. Clinical features of patients infected with 2019 novel coronavirus in Wuhan, China. Lancet. 2020;395(10223):497-506. doi: 10.1016/ S0140-6736(20)30183-5 Epub 2020 Jan 24. Erratum in: Lancet. 2020 Jan 30. PMID: 31986264; PMCID: PMC7159299.

17.Wang D, Hu B, Hu C, Zhu F, Liu X, Zhang J, et al. Clinical Characteristics of 138 Hospitalized Patients With 2019 Novel CoronavirusInfected Pneumonia in Wuhan, China. J Am Med Assoc. 2020;323(11):1061-9. https:// doi.org/10.1001/jama.2020 
18. Zhang ZL, Hou YL, Li DT, Li FZ. Laboratory findings of COVID-19: a systematic review and metaanalysis. Scandinavian J Clinic Lab Invest. 2020;80 (6):441-7. DOI: 10.1080/00365513.2020.1768587

19. Conti P, Ronconi G, Caraffa A, Gallenga CE, Ross R, Frydas I, et al Induction of pro-inflammatory cytokines (IL-1 and IL-6) and lung inflammation by Coronavirus-19 (COVID 19 or SARS-CoV-2): antiinflammatory strategies. J Biol Regul Homeost Agents. 2020;34(2):327-31. doi: 10.23812/CONTI-E PMID: 32171193.

20. Wang L. C-reactive protein levels in the early stage of COVID-19. Med Mal Infect. 2020;50(4):332-34. doi:10.1016/j.medmal.2020.03.007

21. Banerjee D, Popoola J, Shah S, Ster IC, Quan V, Phanish M. COVID-19 infection in kidney transplant recipients. Kidney Int. 2020;97(6):1076-82. doi:10.1016/j.kint.2020.03.018

22. Guo W, Li M, Dong Y, Zhou H, Zhang Z, Tian C, et al. Diabetes is a risk factor for the progression and prognosis of COVID-19. Diabetes Metab Res Rev. 2020;e3319. doi: 10.1002/dmrr.3319 Epub ahead of print. PMID: 32233013; PMCID: PMC7228407

23. Kappert K, Jahić A, Tauber R. Assessment of serum ferritin as a biomarker in COVID-19: bystander or participant? Insights by comparison with other infectious and non-infectious diseases. Biomarkers. 2020;25(8):616-25.

10.1080/1354750X.2020.1797880 Epub 2020 Nov 24. PMID: 32700561.

24. Colafrancesco S, Alessandri C, Conti F, Priori R. COVID-19 gone bad: A new character in the spectrum of the hyperferritinemic syndrome? Autoimmun Rev. 2020;19(7):102573. DOI: 10.1016/ j.autrev.2020.102573

25. Rosário C, Zandman-Goddard G, Meyron-Holtz EG, D'Cruz DP, Shoenfeld Y. The hyperferritinemic syndrome: macrophage activation syndrome, Still's disease, septic shock and catastrophic antiphospholipid syndrome. BMC Med. 2013;11:185. doi: 10.1186/1741-7015-11-185 PMID: 23968282; PMCID: PMC3751883.

26. Olson JD. D-dimer: An Overview of Hemostasis and Fibrinolysis, Assays, and Clinical Applications. Adv Clin Chem. 2015;69:1-46. doi: 10.1016/ bs.acc.2014.12.001. Epub 2015 Feb 7. PMID: 25934358.

27. Yao Y, Cao J, Wang Q, Shi Q, Liu K, LuoZ,et al. Ddimer as a biomarker for disease severity and mortality in COVID-19 patients: a case control study. J Intensive Care. 2020;8:49. doi: 10.1186/s40560-02000466-z PMID: 32665858; PMCID: PMC7348129.
28. Khatri P, Agrawal KK, Sharma D, Chhetri P, Neupane A, Piryani RM, et al. Prevalance of Elevated D-dimer Levels in Confirmed COVID-19 Cases in Intensive Care Unit of a Tertiary Care Centre of Western Nepal. J Nep Med Assoc. 2021;59(235):243-7. doi.org/10.31729/jnma.6284

29. Chen CY, Lee CH, Liu CY, Wang JH, Wang LM, Perng RP. Clinical features and outcomes of severe acute respiratory syndrome and predictive factors for acute respiratory distress syndrome. J Chin Med Assoc. 2005;68(1):4-10. doi: 10.1016/S1726-4901 (09)70124-8 PMID: 15742856; PMCID: PMC7129615.

30. Martinez-Outschoorn UE, Prisco M, Ertel A, Tsirigos A, Lin Z, Pavlides S, et al. Ketones and lactate increase cancer cell "stemness," driving recurrence, metastasis and poor clinical outcome in breast cancer: achieving personalized medicine via MetaboloGenomics. Cell Cycle. 2011;10(8):1271-86. doi: 10.4161/cc.10.8.15330 PMID: 21512313; PMCID: PMC3117136.

31. Henry BM, Aggarwal G, Wong J, Benoit S, Vikse J, Plebani M, et al. Lactate dehydrogenase levels predict coronavirus disease 2019 (COVID-19) severity and mortality: A pooled analysis. Am J Emerg Med. 2020;38(9):1722-26. doi: 10.1016/ j.ajem.2020.05.073 Epub 2020 May 27. PMID: 32738466; PMCID: PMC7251362.

32. Li C, Ye J, Chen Q, Hu W, Wang L, Fan Y, et al. Elevated Lactate Dehydrogenase (LDH) level as an independent risk factor for the severity and mortality of COVID-19. Aging (Albany NY). 2020;12 (15):15670-81. doi: 10.18632/aging.103770 Epub 2020 Aug 14. PMID: 32805722; PMCID: PMC7467395.

33. Martha JW, Wibowo A, Pranata R Prognostic value of elevated lactate dehydrogenase in patients with COVID-19: a systematic review and meta-analysis. Postgrad Med J 2021;0:1-6. doi: 10.1136/ postgradmedj-2020-139542 PMID: 33452143 ; PMCID: PMC7813054

34. Poggiali E, Zaino D, Immovilli P, Rovero L, Losi G, Dacrema A, et al. Lactate dehydrogenase and Creactive protein as predictors of respiratory failure in CoVID-19 patients. Clin Chim Acta. 2020;509:135-8. doi: 10.1016/j.cca.2020.06.012 Epub 2020 Jun 9. PMID: 32531257; PMCID: PMC7282743.

35. Guyi Wang, Chenfang Wu, Quan Zhang, Fang Wu, Bo Yu, JianleiLv, et al. C-Reactive Protein Level May Predict the Risk of COVID-19 Aggravation. Open Forum Infect Dis. 2020;7(5):1-5. https://doi.org/10.1093/ofid/ofaa153

36. Wang L. C-reactive protein levels in the early stage of COVID-19. Méd Maladies Infect. 2020;50 $(4):, 332-4$. j.medmal.2020.03.007 